\title{
Clinical Outcomes and Cardiovascular-Related Events in Young Diabetic Patients with Acute Coronary Syndrome
}

\author{
Mohamad Jarrah', Nasr Alrabadi $\mathbb{D}^{2}$, Nashmi Al-Nusair (D) ${ }^{1}$, Karem H Alzoubi $\mathbb{D}^{3,4}$, Qasim Mhaidat', \\ Mahasen Al-Najar ${ }^{5}$, Ayman Hammoudeh (D) $^{6}$ \\ 'Division of Cardiology, Department of Internal Medicine, Faculty of Medicine, Jordan University of Science and Technology, Irbid, 22I I0, Jordan; \\ ${ }^{2}$ Department of Pharmacology, Faculty of Medicine, Jordan University of Science and Technology, Irbid, 22I I0, Jordan; ${ }^{3}$ Department of Pharmacy \\ Practice and Pharmacotherapeutics, University of Sharjah, Sharjah, United Arab Emirates; ${ }^{4}$ Department of Clinical Pharmacy, Faculty of Pharmacy, \\ Jordan University of Science and Technology, Irbid, Jordan; ${ }^{5}$ Department of Radiology, Faculty of Medicine, The University of Jordan, Amman, Jordan; \\ ${ }^{6}$ Cardiology Department, Istishari Hospital, Amman, Jordan
}

Correspondence: Mohamad Jarrah; Nasr Alrabadi, Tel +962795994247, Email jarrahmohammadI6@gmail.com; nnalrabadi@just.edu.jo

Background: Diabetes mellitus (DM) is considered a risk and prognostic factor for elderly patients with the acute coronary syndrome (ACS). However, less is known about the characteristics of this relationship among younger patients in the Middle Eastern population. Objective: To explore the Clinical outcomes of cardiovascular-related events in young DM patients ( $\leq 50$ years of age) during hospitalization and up to one year of follow-up.

Methods: We compared the clinical features, in-hospital and one-year outcomes in young ACS diabetic patients with young ACS non-diabetic patients in 12 tertiary care centers in Jordan.

Results: A total of 3517 ACS patients were included initially, 1031 of whom (29.3\%) were $<50$-year-old. Of those, 385 (37.3\%) had DM. Compared with young non-diabetic patients, young diabetic patients were more likely to have a worse clinical baseline profile, which includes hypertension ( $53.2 \%$ vs $26.0 \%, \mathrm{p}<0.001)$, dyslipidemia $(32.5 \%$ vs $19.5 \%, \mathrm{p}<0.001)$, and multivessel coronary artery disease $(40.9 \%$ vs $32.0 \%, p=0.03)$. Furthermore, the diabetic group had more females than males $(14.0 \%$ vs $5.9 \%, p<0.001)$ and a higher mean body mass index $(28.8+4.7 \mathrm{~kg} / \mathrm{m} 2 \mathrm{vs} 27.9+4.0 \mathrm{~kg} / \mathrm{m} 2, \mathrm{p}<0.001)$. There were no differences between the two groups in the mortality rates during index hospitalization $(0.39 \%$ vs $0.28 \%, \mathrm{p}=0.63)$ or at one-year follow-up $(1.6 \% \mathrm{vs} 0.6 \%, \mathrm{p}=0.41)$. Also, no significant differences were observed in the rates of stent thrombosis $(1.95 \%$ vs $1.97 \%, \mathrm{p}=0.78)$ or readmission for ACS or coronary revascularization $(10.8 \%$ vs $7.6 \%, \mathrm{p}=0.14)$.

Conclusion: Despite a worse baseline clinical profile, young diabetic ACS patients did not have higher risks for in-hospital or oneyear adverse cardiovascular events compared with the young non-diabetic ACS patients.

Keywords: diabetes mellitus, acute coronary syndrome, cardiovascular events, in-hospital complications, one-year follow-up

\section{Introduction}

Diabetes Mellitus (DM) is a chronic metabolic disease compromising the body's inability to secrete enough insulin, which plays a vital role in regulating blood glucose in the body. ${ }^{1}$ The American Diabetes Association recognizes the following are regarded as risk factors leading to DM, and these include previous family history, $\geq 45$ years of age, overweight, prolonged physical inactivity, low high-density lipoprotein or high triglycerides levels, elevated blood pressure, or those on anti-hypertensive medication, pregnancy and polycystic ovary syndrome. ${ }^{2}$ Many factors cause DM to become identified as an epidemic in many countries around the world, specifically the Middle East. According to the International Diabetes Federation, diabetes is currently affecting over 463 million people as of 2019, 55 million of which reside in the Middle East and is projected to reach 108 million by $2045 .{ }^{3}$ The current trends of DM in the Middle East have started to affect 1 in 8 people especially since the Middle East is composed of developing nations wherein they 
face a leap in socioeconomic growths, prevailing physical inactivity, and their genetic profiles may coincide with the aforementioned risk factors. ${ }^{4,5}$

While DM has a direct relationship with increasing age, recent studies show that similar patterns are widespread even amongst younger strata and the most commonly affected age group is getting younger [Now most commonly among the 40-59 age group]. ${ }^{3}$ With that said, DM poses a major risk of mortality with drastic repercussions. Moreover, it could potentially lead to complications in most body organs including the cardiovascular-related organs promoting the development of Acute Coronary Syndrome (ACS). In fact, according to the International Diabetes Federation, DM patients are $21 \%-32 \%$ prone to developing any cardiovascular disease, and the earlier the onset of DM type II the worse the cardiovascular disease prognosis.,

Since insulin is an essential inflammation modulator whereas hyperglycemia is a pro-inflammatory condition, they both act on increasing fibrinogen in DM patients. ${ }^{7}$ Fibrinogen is an essential factor in the coagulation cascade and when elevated, it poses a risk of becoming a precedent in the causality of ACS and promoting worse outcomes. In the long term and in-hospital stays, notably, fibrinogen is in direct relationship with DM and is a major contributor to cardiovascularrelated morbidity and mortality, which represents the cause for more than $50 \%$ of deaths among DM patients. ${ }^{3,8,9}$ From a therapeutic perspective, uncontrolled hyperglycemia causes glycation of fibrinogen, modifying it into becoming a lysisresistant product when compared to clots in non-diabetic. ${ }^{10}$

Moreover, both low insulin and hyperglycemia promote ACS incidences in many other ways. For instance, insulin may have many cardiovascular protective properties that include the induction of vasodilation and the antagonizing of platelet adhesion. Therefore, low insulin levels prompt small-scale inflammations. Hyperglycemia on the other hand is associated with many risk factors that include hypertension, dyslipidemia, multiple coronary artery diseases, and other factors all of which contribute to ACS.

In addition to the DM being a major detrimental factor in the ACS prognosis, the patterns of affecting the younger strata more than ever before substantiate the necessity to explore the cardiovascular repercussions and the clinical biomarkers coinciding with young DM patients. This is critical given that the long duration of DM can increase the risk of developing cardiovascular disease (CAD) and its associated deaths. ${ }^{12}$ Therefore, this study is concerned with evaluating the impact of cardiovascular-related events in young DM patients $[\leq 50$ years of age] during hospitalization and up to one year of follow-up.,11

\section{Materials and Methods}

\section{Study Design}

A retrospective observational cohort study is based on outcomes derived from the Jordanian PCI registry (JoPRC1); a multi-center Jordanian registry. The protocol of the current study complies with the Declaration of Helsinki. The Institutional Review Board (IRB) at the Jordan University of Science and Technology (IRB-JUST) approved this study. Participants gave informed consent before they participated in the forum.

\section{Study Participants}

Eligible patients followed a consecutive case series whose profiles confirm their diagnosis with ACS and had a prior percutaneous coronary intervention [PCI] procedure done throughout 12 tertiary care centers in Jordan between January 2013 and February 2014.

This study is mainly concerned with revealing the impact of DM on the long and short outcomes of individuals with ACS and aged 50 years or less. The patients were categorized into two groups: one group is diabetic, and the other group is non-diabetic. Their registered records in the JoPRC1 were documented using a case report form during their in-hospital stay and were consented to be followed up for one year. This follow-up protocol was achieved using the patients' continuous health evaluation visits to their respective clinics or phone calls. Other clinical features considered for appraising besides DM were as follows: Hypertension, dyslipidemia, body mass index [BMI], stent thrombosis, major bleeding events, and multi-vessel coronary artery disease. 


\section{Inclusion Criteria and Assessments}

The recruited patients were adults who were $<50$ years of age at the time of recruitment and were diagnosed with an ACS/Stable coronary disease or ACS patients who had PCI. It should be noted that the ACS was defined as patients with acute ST-segment elevation myocardial infarction (STEMI) or non-STEMI and unstable angina.

The DM patients were identified as follows: either previously diagnosed and on anti-diabetic medication or had at least one of the following:

1. At least a test result of $\geq 126 \mathrm{mg} / \mathrm{dL}$ for fasting serum glucose.

2. At least a test result of $\geq 200 \mathrm{mg} / \mathrm{dL}$ 2-hour for a random glucose level.

3. At least a test result of $\geq 6.5 \%$ for $\mathrm{HbAlc}$.

The hypertensive patients were recognized either by being previously diagnosed and on anti-hypertensive medication or those who had a blood pressure measurement elevated past the 140/90 mmHg mark during hospitalization. As well, the patients with dyslipidemia/hypercholesterolemia were recognized as either previously diagnosed and on lipid-lowering agents or had a $>240 \mathrm{mg} / \mathrm{dL}$ total blood cholesterol level. Moreover, the BMI was calculated based on the total body mass $(\mathrm{Kg})$ measurement divided by the body height $\left(\mathrm{m}^{\wedge} 2\right)$ formula.

The other markers or clinical features such as stent thrombosis and major bleeding events had been illustrated based on the standardized definitions of the Academic Research Consortium and CRUSADE, respectively. Finally, the resultant cardiac mortality was defined as any distinct cardiac death source. ${ }^{13}$ Patients who had unclear records or who were not followed properly as planned were excluded from the study.

\section{Statistical Analysis}

The statistical analysis has been performed through the utilization of SPSS statistical software (Version 17, SPSS Inc, Chicago, IL). Statistics are expressed by frequencies and percentages for categorical variables and as mean \pm standard deviation for continuous variables. Two-sided $t$-test and Chi-square test were used in the contrasting efforts between the two groups and a P-value of $<0.05$ was set as the statistically significant value.

\section{Results}

\section{Sample Characteristics}

The data for this study were obtained from 1031 young patients according to the predefined criteria, and these patients were divided into two subgroups based on their DM statuses; one group for those patients with DM (385) and those without DM (646). These two groups were then compared according to their clinical features, in-hospital events, and their outcomes after one year as shown in Table 1. Of worth mentioning, out of the overall 3517 patients included in the registry, 1031 [29.3\%] were $\leq 50$ years old and were eligible for the targeted age group of this study.

\section{Diabetic ACS Patients Compared to Non-Diabetic}

The two groups both of which had ACS consisted of 385 [37.3\%] diabetic and 464 [62.7\%] non-diabetic patients; females made up 54 [14.0\%] and 38 [5.9\%] of each group, respectively, which happens to be significantly different $[<0.001]$.

The results obtained showed that the following clinical baseline variables were statistically significant by which young diabetic ACS patients were more liable to have hypertension [53.2\% vs $26.0 \%$, p $<0.001$ ], dyslipidemia [32.5\% vs $19.5 \%, \mathrm{p}<0.001$ ], multivessel coronary artery disease [ $40.9 \%$ vs $32.0 \%, \mathrm{p}=0.03$ ], higher body mass index $(28.8$ $+4.7 \mathrm{~kg} / \mathrm{m}^{2}$ vs $27.9+4.0 \mathrm{~kg} / \mathrm{m}^{2}, \mathrm{p}<0.001$ ) in relation to non-diabetic ACS patients with smoking being the only marker that is higher in non-diabetics [61.3\% vs $72 \%, \mathrm{p}<0.001]$. Notably though, the STEMI was higher in non-diabetics $[39.9 \% \mathrm{vs} 48.7 \%, \mathrm{p}=0.02]$. With that stated, henceforth the rest of the markers such as age, previous family history, and mean GFR were not significantly different and so in-hospital events and one-year outcomes were also not significantly different; so the mortality rate during hospitalization $[0.39 \%$ vs $0.28 \%, \mathrm{p}=0.63]$ at one year $(1.6 \% \mathrm{vs} 0.6 \%, \mathrm{p}=0.41)$, 
Table I Baseline Features for Young Patients with and without DM

\begin{tabular}{|c|c|c|c|}
\hline Features & $\begin{array}{l}\text { Young DM } \\
\text { [385 (37.3\%)] }\end{array}$ & $\begin{array}{l}\text { Young Non-DM } \\
\text { [646 (62.7\%)] }\end{array}$ & $P$ value \\
\hline \multicolumn{4}{|l|}{ Demographic Baseline Features } \\
\hline Mean age $Y$ & $43.2+5.3$ & $43.0+5.5$ & 0.56 \\
\hline$\leq 40 \mathrm{Y}$ & $66[17.1 \%]$ & $139[21.5 \%]$ & 0.1 \\
\hline Females & $54[14.0 \%]$ & $38[5.9 \%]$ & $<0.001$ \\
\hline Hypertension & $205[53.2 \%]$ & $168[26.0 \%]$ & $<0.001$ \\
\hline Smokers & $236[61.3 \%]$ & 465 [72\%] & $<0.001$ \\
\hline Dyslipidimia & $125[32.5 \%]$ & $126[19.5 \%]$ & $<0.001$ \\
\hline Premature CVD family $\mathrm{Hx}$ & $213[55.3 \%]$ & $350[54.2 \%]$ & 0.78 \\
\hline Mean BMI & $28.8+4.7$ & $27.9+4.0$ & 0.001 \\
\hline Mean GFR & $128+38.7$ & $127.6+30.8$ & 0.71 \\
\hline \multicolumn{4}{|c|}{ Disease and Coronary Artery Disease [CAD] } \\
\hline & Of JOPCR I Patients 257 & Of JOPCRI Patients 356 & \\
\hline STEMI & $123[39.9 \%]$ & $231[48.7 \%]$ & 0.02 \\
\hline Multivessel CAD & 257 [40.9\%] & 356 [32\%] & 0.03 \\
\hline ST segment deviation & $59[15.3 \%]$ & $101[15.6 \%]$ & 0.97 \\
\hline Elevated cardiac enzymes & $116[30.1 \%]$ & $196[30.3 \%]$ & 0.99 \\
\hline \multicolumn{4}{|l|}{ In-Hospital Events } \\
\hline Heart failure & $7 \%$ & $6.5 \%$ & 0.94 \\
\hline Stent thrombosis & $0.78 \%$ & $0.84 \%$ & 0.71 \\
\hline Death & $0.39 \%$ & $0.28 \%$ & 0.63 \\
\hline Ventricular tachycardia & $0.07 \%$ & $1.1 \%$ & 0.09 \\
\hline \multicolumn{4}{|l|}{ One Year Events } \\
\hline Stent thrombosis & $1.95 \%$ & $1.97 \%$ & 0.78 \\
\hline Death & $1.56 \%$ & $0.56 \%$ & 0.41 \\
\hline Revascularization or readmission for ACS & $10.8 \%$ & $7.6 \%$ & 14 \\
\hline
\end{tabular}

the rates of stent thrombosis $(1.95 \%$ vs $1.97 \%, \mathrm{p}=0.78)$ or readmission for ACS or coronary revascularization $(10.8 \%$ vs $7.6 \%, \mathrm{p}=0.14)$ were all similar between both groups.

\section{Discussion}

The results of this study indicated that young patients with an age less than 50 years contribute significantly (30\%) to the pool of CAD patients in the Middle Eastern countries and especially in Jordan. Among those, the diabetic patients represent around $40 \%$. Those percentages are considered high among other populations. ${ }^{14,15}$ Furthermore, the results of this study ring the bells toward applying more proper policies to reduce the burden of those diseases at earlier ages.

As expected, our results showed as well that the diabetic patients had a generally worse baseline clinical profile indicating that they may suffer from what had been called the "metabolic syndrome". In other words, they were more prone to have coexistence of other co-morbidities that are considered risk factors for cardiovascular diseases. ${ }^{16}$ More specifically, the cohort of DM patients showed higher blood pressure levels, higher BMI, and more frequent dyslipidemia. Albeit STEMI was more prevalent in diabetics in addition to having a higher incidence of multivessel coronary artery disease, which is considered as the main mortality predictor in diabetics. ${ }^{17}$ Consequently, those risk factors are expected to worsen the patients' clinical outcomes. ${ }^{17,18}$

On the other hand, the mortality rates and the incidence of in-hospital and one-year adverse cardiovascular events in the DM group compared to their counterparts did not change. This may contradict the results obtained from other studies. ${ }^{6,19}$ However, given the young ages of the participants, the effect of those risk factors including DM and their accompanied complications may need more time to significantly affect the prognosis of CAD. ${ }^{6}$ 
Moreover, our results showed that the percentage of females in the diabetic group is higher than that of the nondiabetic. Diabetic females are known to have more risk of developing cardiovascular-related diseases and death risk of about 7.5 times more than non-diabetic females. ${ }^{20}$

DM patients in this study were less likely to be smokers. However, this may be related to the higher percentage of females in the diabetic group (who are known in Jordan to be fewer smokers compared to males). ${ }^{21}$ As well, it could be related to the fact that the patients with baseline comorbidities like DM, hypertension, and dyslipidemia are highly encouraged by their caregivers and highly self-motivated to quit smoking before having CAD and before being recruited to join this study.

Despite these findings in a younger than usual population, the medical literature has proven that DM acts as a consistent major setback to patients who fall victim to it. Adults in other studies were generally found to have a higher mortality ratio compared to non-diabetics due to cardiovascular diseases ${ }^{22}$ and were responsible for over 1.7 million hospitalizations for related cardiovascular diseases in the USA according to the national diabetes statistics report in $2020 .{ }^{23} \mathrm{DM}$ is undoubtedly linked to cardiovascular diseases and mortalities, but the earlier the onset and the longer the follow-up, the higher the risk of developing related cardiovascular diseases. ${ }^{22}$ Patients who had earlyonset diabetes had a higher death expectation rate than those with late-onset as it relies upon the long-term exposure to DM complications and the complications related to the other associated risk factors and comorbidities. ${ }^{22,24}$

\section{Limitations}

Although the ACS patients were followed prospectively in this cohort, however, their DM status was obtained retrospectively. In other words, it was not accurately known for how long they have been diagnosed with DM and if it was controlled or not. As well, although the number of the patients was high considering a multicenter study, however, the number of patients who developed serious outcomes was small even after one year of follow-up.

\section{Conclusions}

Despite a worse baseline clinical profile, young diabetic ACS patients did not have higher risks for in-hospital or oneyear adverse cardiovascular events compared with the young non-diabetic ACS patients. However, this could be related to the short follow-up time of one year considering that the risk for cardiovascular complications in DM patients can increase with time. Therefore, more similar studies with longer follow-up periods or studies that depend on following the DM patients for long durations may be warranted.

\section{Disclosure}

The authors report no conflicts of interest in this work.

\section{References}

1. American Diabetes Association. Diagnosis and Classification of Diabetes Mellitus. Diabetes Care. 2004;27(1):S5-S10. doi:10.2337/diacare.27.2007.S5

2. American Diabetes Association. Diabetes Risk, 2014. Available from: https://www.diabetes.org/diabetes-risk. Accessed January $21,2021$.

3. International Diabetes Federation, IDF Diabetes Atlas. 9th ed. Brussels, Belgium; 2019. Available from: https://www.diabetesatlas.org. Accessed February 11, 2022.

4. Kalan Farmanfarma KH, Ansari-Moghaddam A, Zareban I, Adineh HA. Prevalence of type 2 diabetes in Middle-East: systematic review\& metaanalysis. Prim Care Diabetes. 2020;14(4):297-304. doi:10.1016/j.pcd.2020.01.003

5. Al Busaidi N, Shanmugam P, Manoharan D. Diabetes in the middle east: government health care policies and strategies that address the growing diabetes prevalence in the middle east. Curr Diab Rep. 2019;19:8. doi:10.1007/s11892-019-1125-6

6. Hillier T, Pedula K. Complications in young adults with early-onset type 2 diabetes. Diabetes Care. 2003;26(11):2999-3005. doi:10.2337/ diacare.26.11.2999

7. Abdul Razak MK, Sultan A. The importance of measurement of plasma fibrinogen level among patients with type- 2 diabetes mellitus. Diabetes Metab Syndr. 2019;13(2):1151-1158. doi:10.1016/j.dsx.2019.01.049

8. Dhawale S, Jayant S, Gupta A. Serum fibrinogen level in type 2 diabetes mellitus patients. Int J Adv Med. 2016;3:83-87. doi:10.18203/2349-3933. ijam20160194

9. Müdespacher D, Radovanovic D, Camenzind E, et al. Admission glycaemia and outcome in patients with acute coronary syndrome. Diabetes Vasc Dis Res. 2007;4(4):346-352. doi:10.3132/dvdr.2007.063

10. Pieters M, Van Zyl D, Rheeder P, et al. Glycation of fibrinogen in uncontrolled diabetic patients and the effects of glycaemic control on fibrinogen glycation. Thromb Res. 2007;120(3):439-446. doi:10.1016/j.thromres.2006.10.016 
11. Sun Q, Li J, Gao F. New insights into insulin: the anti-inflammatory effect and its clinical relevance. World J Diabetes. 2014;5(2):89-96. doi:10.4239/wjd.v5.i2.89

12. Fox CS, Sullivan L, D'Agostino RB, Wilson PWF. The significant effect of diabetes duration on coronary heart disease mortality: the Framingham heart study. Diabetes Care. 2004;27(3):704-708. doi:10.2337/diacare.27.3.704

13. Cutlip DE, Windecker S, Mehran R, et al. The academic research consortium. Clinical end points in coronary stent trials: a case for standardized definitions. Circulation. 2007;115(17):2344-2351. doi:10.1161/CIRCULATIONAHA.106.685313

14. Al-Shudifat AE, Johannessen A, Azab M, et al. Risk factors for coronary artery disease in patients undergoing elective coronary angiography in Jordan. BMC Cardiovasc Disord. 2017;17(183). doi:10.1186/s12872-017-0620-4.

15. Al-Nozha MM, Ismail HM, Al Nozha OM. Coronary artery disease and diabetes mellitus. J Taibah Univ Med Sc. 2016;11(4):330-338.

16. American Heart Association. About Metabolic Syndrome;2021. Available from: https://www.heart.org/en/health-topics/metabolic-syndrome/aboutmetabolic-syndrome. Accessed October 20, 2021.

17. Ford E, DeStefano F. Risk factors for mortality from all causes and from coronary heart disease among persons with diabetes: findings from the national health and nutrition examination survey I Epidemiologic follow-up study. Am J Epidemiol. 1991;133(12):1220-1230. doi:10.1093/ oxfordjournals.aje.a115834

18. Mahalle N, Garg M, Naik S. Association of metabolic syndrome with severity of coronary artery disease. Indian J Endocrinol Metab. 2014;18 (5):708-714. doi:10.4103/2230-8210.139238

19. Kanaya A, Grady D, Barrett-Connor E. Explaining the sex difference in coronary heart disease mortality among patients with Type 2 diabetes mellitus. Arch Intern Med. 2002;162(15):1737-1745. doi:10.1001/archinte.162.15.1737

20. Cooper S, Caldwell J. Coronary artery disease in people with diabetes: diagnostic and risk factor evaluation. Clin Diabetes. 1999;17(2):58-70.

21. Jaghbir M, Shreif S, Ahram M. Pattern of cigarette and waterpipe smoking in the adult population of Jordan. Eastern Med Health J. 2014;20 (9):529-537. doi:10.26719/2014.20.9.529

22. Centers for disease control and prevention. Diabetes and your heart; 2020. Available from: https://cdc.gov. Accessed February $26,2021$.

23. Centers for Disease Control and Prevention. National Diabetes Statistics Report, 2020. Atlanta, GA: Centers for Disease Control and Prevention, U.S. Dept of Health and Human Services; 2020. Available from: https://cdc.gov. Accessed February 11, 2022.

24. Huo X, Gao L, Guo L, et al. Risk of non-fatal cardiovascular diseases in early-onset versus late-onset type 2 diabetes in China: a cross-sectional study. Lancet Diabetes Endocrinol. 2016;4(4):115-124. doi:10.1016/S2213-8587(15)00508-2

\section{Publish your work in this journal}

Vascular Health and Risk Management is an international, peer-reviewed journal of therapeutics and risk management, focusing on concise rapid reporting of clinical studies on the processes involved in the maintenance of vascular health; the monitoring, prevention and treatment of vascular disease and its sequelae; and the involvement of metabolic disorders, particularly diabetes. This journal is indexed on PubMed Central and MedLine. The manuscript management system is completely online and includes a very quick and fair peer-review system, which is all easy to use. Visit http://www.dovepress.com/testimonials.php to read real quotes from published authors. 